\title{
A CANTOR FUNCTION CONSTRUCTED BY CONTINUED FRACTIONS
}

\author{
F. HERZOG AND B. H. BISSINGER
}

1. Introduction. The well known Cantor function was treated in a paper by Hille and Tamarkin [5 $]^{1}$, who collected together a few properties of that function, which is often used as an example in the theory of functions of a real variable. In the definition of this function, the representation of the numbers of the closed interval $[0,1]$ in the arithmetic scales of radices 3 and 2 (ternary and binary scales of notation) was used. Gilman [3] generalized the above function by replacing the radices 3 and 2 by $\alpha$ and $\beta$, respectively, where $\alpha$ and $\beta$ are integers such that $\alpha>\beta>1$ and $\alpha-1$ is divisible by $\beta-1$. He thus obtained a whole class of functions of the Cantor type.

The present paper gives an example of a function of the same type, based on the representation of numbers as simple continued fractions. While in the main our function has the same properties as those treated in [5] and [3], there are also some interesting differences between them, to some of which we shall call attention as the occasion arises. Since some of the results of [5] are a special case of those of [3] (with $\alpha=3, \beta=2$ ), it will in most cases suffice to compare our results with those of $[3]$.

2. The set $E$. Let $0<x<1$. If the positive integers $a_{1}, a_{2}, a_{3}, \cdots$ are successively the denominators (partial quotients) appearing in the expansion of $x$ in a simple continued fraction, we shall write

$$
x=\frac{1}{a_{1}}+\frac{1}{a_{2}}+\frac{1}{a_{3}}+\cdots=\left\{a_{1}, a_{2}, a_{3}, \cdots\right\} .
$$

In the case of a rational $x, 0<x<1$, that is, of a finite simple continued fraction, we have two such expansions, namely, $x=\left\{a_{1}, \cdots, a_{n-1}, a_{n}\right\}$ $=\left\{a_{1}, \cdots, a_{n-1}, a_{n}-1,1\right\}$, where $a_{n} \geqq 2$. Both of these expansions will be admitted in this paper, also the expansion $1=\{1\}$.

Throughout the paper we shall use the letter $c$ (with or without subscript) to denote positive integers.

We now define the set $E$ as the set consisting of $x=0$ and of all $x$, $0<x<1$, which can be expanded in a simple continued fraction none of whose denominators equals unity. In other words, $E$ contains $x=0$ and all finite and infinite simple continued fractions of the form

Received by the editors May 3, 1946.

${ }^{1}$ Numbers in brackets refer to the bibliography at the end of the paper. 
$x=\left\{c_{1}+1, c_{2}+1, \cdots\right\}$. The above wording is to imply that $x$ $=\left\{c_{1}+1, \cdots, c_{n}+1\right\}$ does belong to $E$ although it can also be written as $x=\left\{c_{1}+1, \cdots, c_{n}, 1\right\}$.

The set complementary to $E$ with respect to the interval $[0,1]$ will be denoted by $D$. Thus $D$ consists of $x=1$ and of all open intervals with end points $\xi$ and $\xi^{\prime}$, where ${ }^{2}$

$$
\begin{aligned}
\xi & =\left\{c_{1}+1, c_{2}+1, \cdots, c_{n-1}+1,1\right\} \\
& =\left\{c_{1}+1, c_{2}+1, \cdots, c_{n-1}+2\right\}, \\
\xi^{\prime} & =\left\{c_{1}+1, c_{2}+1, \cdots, c_{n-1}+1,2\right\} .
\end{aligned}
$$

Such an interval will be called a $D_{n}$-interval in the following. (For examples of $D_{n}$-intervals, see the table at the end of the paper.) There is one $D_{1}$-interval, $(1 / 2,1)$, but infinitely many $D_{n}$-intervals for each $n \geqq 2$. It is easily seen that any two $D_{n}$-intervals (with equal or unequal values of $n$ ) do not overlap and do not even have an end point in common.

The points of $E$ (except for $x=0$ ) can be divided into two classes, $E_{R}$ and $E_{I} . E_{R}$ is to contain the rational $x \in E$, other than zero, and $E_{I}$ the irrational $x \in E$. It is clear from the above that $x \in E_{R}$ if and only if $x$ is the end point of a $D_{n}$-interval (except $x=1$, which does not belong to $E$ at all). $x=0$ will be considered as belonging neither to $E_{R}$ nor to $E_{I}$.

We shall now derive a few of the main properties of the set $E$.

(2.1) $E$ is a closed set.

Consider $D$ as the union of the interval $1 / 2<x \leqq 1$ and all open $D_{n}$-intervals, $n=2,3, \cdots$. All these intervals are open sets with respect to $[0,1]$. Thus $D$ is an open set and $E$ is a closed one.

(2.2) $E$ is a perfect set.

The following three relations, referring to the cases $x=0, x \in E_{R}$, $x \in E_{I}$, respectively, show that $E$ is perfect. (In each case $m \rightarrow+\infty$ through integral values.)

$$
\begin{aligned}
0 & =\lim _{m}\{m\}, \\
\left\{c_{1}+1, c_{2}+1, \cdots, c_{n}+1\right\} & =\lim _{m}\left\{c_{1}+1, c_{2}+1, \cdots, c_{n}+1, m\right\}, \\
\left\{c_{1}+1, c_{2}+1, \cdots\right\} & =\lim _{m}\left\{c_{1}+1, c_{2}+1, \cdots, c_{m}+1\right\} .
\end{aligned}
$$

2 If $n=1$ the numbers in (1) are to be interpreted as meaning $\xi=\{1\}=1$ and $\xi^{\prime}=\{2\}=1 / 2$. We also remark that $\xi<\xi^{\prime}\left(\xi>\xi^{\prime}\right)$ when $n$ is even (odd). See [8, p. 41, Theorem 8]. 
(2.3) E has the power of the continuum.

$E$ contains as a subset the set of all infinite simple continued fractions $\left\{c_{1}+1, c_{2}+1, \cdots\right\}$ with each $c_{i}=1$ or 2 .

(2.4) $E$ is of Lebesgue measure zero.

This is a result due to F. Bernstein $[1$, p. 429 , Remark]. (See also $[4$, p. 334, corollary $]$.)

(2.5) $E$ is nowhere dense in $[0,1]$.

The complementary set $D$ is by (2.4) of measure one, hence everywhere dense in $[0,1]$. It is also open in that interval by (2.1). Hence (see $[2$, p. 64, definition $]$ ) $E$ is nowhere dense in $[0,1]$.

Comparing our set $E$ with the corresponding sets $P_{\alpha \beta} \equiv P$ of [3], we see that they agree in the five properties, derived above. The subsets $E_{R}$ and $E_{I}$ of $E$ correspond, respectively, to the subsets $P^{-}+P^{+}$and $P^{0}$ of $P$. (See [3, p. 434].) However, while $P$ is symmetric about $x=1 / 2, E$ has no such symmetry. A more essential difference is that the intervals $\delta_{p k}$ of $[5$, p. 255] for fixed $p$ are finite in number and of equal length, while in our case we have infinitely many $D_{n}$-intervals for fixed $n \geqq 2$ and their lengths are not equal.

3. Definition and main properties of $\phi(x)$. We shall first define our function for $x \in E$ by the following three equations, which apply to the cases $x=0, x \in E_{R}, x \in E_{I}$, respectively.

$$
\begin{gathered}
\phi(0)=0 . \\
\text { (2a) } x=\left\{c_{1}+1, c_{2}+1, \cdots, c_{n}+1\right\}: \phi(x)=\left\{c_{1}, c_{2}, \cdots, c_{n}\right\} . \\
\text { (2c) } x=\left\{c_{1}+1, c_{2}+1, \cdots\right\}: \phi(x)=\left\{c_{1}, c_{2}, \cdots\right\} .
\end{gathered}
$$

Before extending this definition to values of $x \in D$, we first define $\phi(1)=1$ and show that the values of $\phi(x)$ at both end points of each $D_{n}$-interval are equal to one another. This is true for the $D_{1}$-interval $(1 / 2,1)$, since $\phi(1 / 2)=\phi(\{2\})=\{1\}=1=\phi(1)$, and for a $D_{n}$-interval with $n \geqq 2$ we see from (2b) that, when $\xi$ and $\xi^{\prime}$ are given by (1), we have

$$
\phi(\xi)=\left\{c_{1}, c_{2}, \cdots, c_{n-1}+1\right\}=\left\{c_{1}, c_{2}, \cdots, c_{n-1}, 1\right\}=\phi\left(\xi^{\prime}\right) .
$$

We are now able to define $\phi(x)$ for any interior point of a $D_{n}$-interval as the common value of $\phi(x)$ at the end points of that interval. (For examples, see the table at the end of the paper.) The definition of $\phi(x)$ in $[0,1]$ is thus complete.

We shall now derive some of the more important properties of $\phi(x)$.

(3.1) $\phi(x)$ is constant in every (closed) $D_{n}$-interval. The union of the intervals of constancy of $\phi(x)$ is a set of measure one. 
This follows at once from the definition of $\phi(x)$ for $x \in D$ and from (2.4).

(3.2) $\phi(x)$ is a nondecreasing function of $x$ in $[0,1]$; moreover, when $0 \leqq x<x^{\prime} \leqq 1$ then $\phi(x) \leqq \phi\left(x^{\prime}\right)$, the equality holding if and only if $x$ and $x^{\prime}$ lie in the same (closed) $D_{n}$-interval.

On account of (3.1), it suffices to consider the case in which $x$ and $x^{\prime}$ belong to $E$. Since by (2a) the case $x=0$ is trivial we may assume furthermore that $0<x<x^{\prime} \leqq 1 / 2$. Now let $x$ and $x^{\prime}$ be written in the form (2b) or (2c) and assume that they agree in the first $m-1$ denominators, but no further $(m \geqq 1)$. Then when $m$ is even (odd) either the $m$ th denominator of $x^{\prime}$ is larger (smaller) than that of $x$, or $x^{\prime}(x)$ has only $m-1$ denominators. (See $[8$, pp. 40-41, Theorems 7 and 8].) The definitions (2b) and (2c) of $\phi(x)$ are such that these same relations will hold true for the simple continued fractions for $\phi(x)$ and $\phi\left(x^{\prime}\right)$, so that in general $\phi(x)<\phi\left(x^{\prime}\right)$. And it is easily seen that the only exceptional case, leading to $\phi(x)=\phi\left(x^{\prime}\right)$, is that in which $x$ and $x^{\prime}$ are the smaller and larger, respectively, of the numbers $\xi$ and $\xi^{\prime}$ (with $n \geqq 2$ ) of (1). This completes the proof.

(3.3) The function $y=\phi(x)$ assumes every value of $y$ of the interval $0 \leqq y \leqq 1$. Moreover, if $y$ is rational and $0<y \leqq 1$ then $y$ is assumed by $\phi(x)$ in one whole $D_{n}$-interval, while $y=0$ and every irrational $y$, $0<y<1$, is assumed at one point $x$.

This follows at once from the definitions (2a), (2b) and (2c) of the function $\phi(x)$ and its definition for $x \in D$.

(3.4) $\phi(x)$ is continuous in $[0,1]$.

A function $f(x)$ which is defined and monotone in a closed interval $[a, b]$ is continuous in that interval if and only if it assumes every value between $f(a)$ and $f(b)$. Thus (3.4) follows from (3.2) and (3.3).

(3.5) For every positive $\lambda$, the $\lambda$-variation (see [2, p. 511, Definition]) of $\phi(x)$ in $[0,1]$ is equal to unity; $\phi(x)$ is not absolutely continuous.

We shall show the first part of (3.5) by proving the more general theorem below, whose hypotheses are satisfied for $\phi(x)$ by (3.2) and (3.1). The second part of (3.5) follows from the first, but could be directly concluded from the fact that $\int_{0}^{1} \phi^{\prime}(x) d x=0$, but $\phi(1)-\phi(0)=1$.

THEOREM 1. If $f(x)$ is monotone $e^{3}$ in the closed interval $[a, b]$ and if the intervals of constancy of $f(x)$ cover $[a, b]$ except for a set of measure zero, then the $\lambda$-variation of $f(x)$ in $[a, b]$ equals $|f(b)-f(a)|$ for every positive $\lambda$.

Let $f(x)$ be nondecreasing. (If $f(x)$ is nonincreasing consider the

\footnotetext{
${ }^{3}$ Continuity of $f(x)$ is not assumed.
} 
function $-f(x)$.) Let $\lambda>0$ be given. We denote the intervals of constancy of $f(x)$, of which there are at most denumerably many, in some order by $I_{i}(j=1,2, \cdots)$ and their lengths by $\left|I_{i}\right|$. Since $\sum_{j}\left|I_{j}\right|=b-a$ we can in any case choose $k$ such that $\sum_{j=1}^{k}\left|I_{j}\right|>b$ $-a-\lambda$. We now replace these $k$ intervals $I_{j}$ by intervals $I_{j}{ }^{*}$ such that the end points of $I_{j}^{*}$ are inner points of $I_{j}(j=1,2, \cdots, k)$ and such that we still have $\sum_{j=1}^{k}\left|I_{j}^{*}\right|>b-a-\lambda$. Let these $k$ intervals $I_{j}^{*}$ be denoted in their natural order (from left to right) by $\left(x_{1}, x_{2}\right),\left(x_{3}, x_{4}\right), \cdots,\left(x_{2 k-1}, x_{2 k}\right)$. We know then that $f\left(x_{2 i-1}\right)=f\left(x_{2 i}\right)$ for $i=1,2, \cdots, k$. The complementary intervals $\left(a, x_{1}\right),\left(x_{2}, x_{3}\right), \cdots$, $\left(x_{2 k-2}, x_{2 k-1}\right),\left(x_{2 k}, b\right)$ have a combined length less than $\lambda$, while (with $\left.x_{0}=a, x_{2 k+1}=b\right)$

$$
\sum_{i=0}^{k}\left[f\left(x_{2 i+1}\right)-f\left(x_{2 i}\right)\right]=f(b)-f(a) .
$$

This completes the proof of Theorem 1.

(3.6) The curve $y=\phi(x)$ is of arc length ${ }^{4}$.

This will be proved by the following theorem. Its hypotheses are satisfied by $\phi(x)$ on account of (3.2) and (3.5).

THEOREM 2. If $f(x)$ is monotone $e^{3}$ in the closed interval $[a, b]$ and if its $\lambda$-variation in $[a, b]$ equals $|f(b)-f(a)|$ for every positive $\lambda$, then the arc length ${ }^{4}$ of the curve $y=f(x)$ is $b-a+|f(b)-f(a)|$.

Let $f(x)$ be nondecreasing. That the curve $y=f(x)$ is rectifiable follows from the fact that $f(x)$ is of bounded variation. Since the length of any inscribed polygon can obviously not exceed $b-a+f(b)-f(a)$ it suffices to show the existence of an inscribed polygon of length greater than $b-a+f(b)-f(a)-\epsilon$ for every given positive $\epsilon$. By the hypothesis, we can select a set of intervals $\left(x_{1}, x_{2}\right),\left(x_{3}, x_{4}\right), \cdots$, $\left(x_{2 h-1}, x_{2 h}\right)$ with $x_{j}<x_{j+1}(j=1,2, \cdots, 2 h-1)$ such that

$$
\begin{aligned}
\sum_{j=1}^{h}\left(x_{2 j}-x_{2 j-1}\right) & <\frac{\epsilon}{2}, \\
\sum_{j=1}^{h}\left[f\left(x_{2 j}\right)-f\left(x_{2 j-1}\right)\right] & >f(b)-f(a)-\frac{\epsilon}{2} .
\end{aligned}
$$

Let $P_{i}$ be the point $\left(x_{i}, f\left(x_{i}\right)\right), i=1,2, \cdots, 2 h$, and form the polygon

${ }^{4}$ If $y=f(x)$ is of bounded variation in $[a, b]$ then the two definitions of arc length by Peano and by Jordan and Scheeffer (see [6, p. 338]) are equivalent for functions $f(x)$ which have the additional property that for each $x, a<x<b$, either $f(x-) \leqq f(x)$ $\leqq f(x+)$ or $f(x-) \geqq f(x) \geqq f(x+)$. (See [7, pp. 300-302].) Monotone functions obviously have this property. The definition used in the proof of Theorem 2 is Peano's definition. 
$P_{0} P_{1} P_{2} \cdots P_{2 h} P_{2 h+1}$, where $P_{0}=(a, f(a))$ and $P_{2 h+1}=(b, f(b))$. By (3) the projections of the segments $P_{0} P_{1}, P_{2} P_{3}, \cdots, P_{2 h-2} P_{2 h-1}, P_{2 h} P_{2 h+1}$ on the $x$-axis have a combined length greater than $b-a-\epsilon / 2$; and by (4) the projections of the segments $P_{1} P_{2}, P_{3} P_{4}, \cdots, P_{2 h-1} P_{2 h}$ on the $y$-axis have a combined length greater than $f(b)-f(a)-\epsilon / 2$. This completes the proof of Theorem 2.

The function $\phi(x)$ has the properties, which were derived in this section, in common with the functions $\omega_{\alpha \beta}(x) \equiv \omega(x)$ of [3]. In fact, properties (3.5) and (3.6) were proved simultaneously for all of these functions by Theorems 1 and 2, respectively. It is to be observed, however, that according to (3.3) our curve $y=\phi(x)$ has all rational numbers $y, 0<y \leqq 1$, as ordinates of its horizontal segments, while the functions $\omega_{\alpha \beta}(x)$ of [3] have as such only certain rational numbers $y, 0<y<1$, namely, those of the form $y=m / \beta^{r}, r$ and $m$ being positive integers and $\beta$ being the radix, used for the values of $y$.

4. The derivatives of $\phi(x)$. The problem of determining the derivatives of the functions $\omega_{\alpha \beta}(x)$ of [3] was dealt with by Gilman [3, pp. 438-442], who obtained conditions for the existence of the derivative on the left and that on the right at the points of the set $P_{\alpha \beta}$. The various possibilities are listed on p. 442 of [3]. The corresponding problem for our function $\phi(x)$ seems to be more difficult. Except for one case (see footnote 7 below), we shall obtain the values of the four derivatives of $\phi(x)$ at all points $x$ of $[0,1]$.

We introduce first the following notations. In this section $x_{0}$ $=\left\{c_{1}+1, c_{2}+1, \cdots\right\}$ will always denote a fixed number such that $x_{0} \in E, x_{0} \neq 0$. If the simple continued fraction for $x_{0}$ has at least $n$ denominators we shall denote the $n$th complete quotient of $x_{0}$ by $c_{n}+1+s_{n}$, where $s_{n}=0$ if $x_{0}$ has only $n$ denominators and otherwise

$$
s_{n}=\left\{c_{n+1}+1, c_{n+2}+1, \cdots\right\} \text {. }
$$

In either case we have $s_{n} \in E$, so that $0 \leqq s_{n} \leqq 1 / 2$, and we conclude from $[8$, p. 27, Rule A] that

$$
x_{0}=\left\{c_{1}+1, c_{2}+1, \cdots, c_{n-1}+1, c_{n}+1+s_{n}\right\} .
$$

It follows readily from (2a), (2b) and (2c) in conjunction with (5) that

$$
\phi\left(x_{0}\right)=\left\{c_{1}, c_{2}, \cdots, c_{n-1}, c_{n}+\phi\left(s_{n}\right)\right\} .
$$

We denote the $m$ th convergent of the simple continued fraction for $x_{0}$ by $P_{m} / Q_{m}$ and that for $\phi\left(x_{0}\right)$ by $^{6} p_{m} / q_{m}(m=1,2, \cdots)$ and obtain

\footnotetext{
${ }^{5}$ If $x_{1}=a$ the polygon is to start with $P_{1}$ and if $x_{2 h}=b$ it is to end with $P_{2 h}$.
}

${ }^{6}$ As usual we put $P_{-1}=p_{-1}=Q_{0}=q_{0}=1$ and $P_{0}=p_{0}=Q_{-1}=q_{-1}=0$. 


$$
\begin{aligned}
x_{0} & =\frac{\left(c_{n}+1+s_{n}\right) P_{n-1}+P_{n-2}}{\left(c_{n}+1+s_{n}\right) Q_{n-1}+Q_{n-2}} ; \\
\phi\left(x_{0}\right) & =\frac{\left[c_{n}+\phi\left(s_{n}\right)\right] p_{n-1}+p_{n-2}}{\left[c_{n}+\phi\left(s_{n}\right)\right] q_{n-1}+q_{n-2}}
\end{aligned}
$$

from (6) and (7). If now $x$ is any number such that $x \in E, x \neq 0, x \neq x_{0}$, and (at least) the first $n-1$ denominators of the simple continued fraction of $x$ agree with those of $x_{0}$ we may write in analogy to (6)

$$
x=\left\{c_{1}+1, c_{2}+1, \cdots, c_{n-1}+1, c+1+s\right\},
$$

where, as in (6), $c$ is a positive integer and $s \in E$, so that $0 \leqq s \leqq 1 / 2$. Formulas analogous to (7) and (8) hold also for $x$. By the use of the formulas (8) for $x_{0}$ and $x$ and the relation $P_{n-1} Q_{n-2}-P_{n-2} Q_{n-1}$ $=p_{n-1} q_{n-2}-p_{n-2} q_{n-1}=(-1)^{n}$ we then obtain the following formula:

$$
\begin{aligned}
\Delta\left(x, x_{0}\right) \equiv & {\left[\phi(x)-\phi\left(x_{0}\right)\right] /\left(x-x_{0}\right) } \\
= & \frac{c-c_{n}+\phi(s)-\phi\left(s_{n}\right)}{c-c_{n}+s-s_{n}} \cdot \frac{\left(c_{n}+1+s_{n}\right) Q_{n-1}+Q_{n-2}}{\left[c_{n}+\phi\left(s_{n}\right)\right] q_{n-1}+q_{n-2}} \\
& \cdot \frac{(c+1+s) Q_{n-1}+Q_{n-2}}{[c+\phi(s)] q_{n-1}+q_{n-2}} .
\end{aligned}
$$

We shall also need the following inequalities. If $x \in E, x \neq 0$, we can write $x$ in the form (9) with $n=1$, that is, $x=\{c+1+s\}$ with positive integral $c$, and $s \in E$; by (7), $\phi(x)=\{c+\phi(s)\}$ and hence

$$
\phi(x) / x=(c+1+s) /[c+\phi(s)],
$$

so that $\phi(x) / x>1$. Therefore, $\phi(x) / x \leqq(c+1+s) /(c+s) \leqq(c+1) / c \leqq 2$. In view of $0 \leqq s \leqq 1 / 2$ we conclude now that $\phi(x) / x \geqq(c+1+s) /(c+2 s)$ $\geqq(c+3 / 2) /(c+1) \geqq 1+1 / 4 c$. We thus obtained for $x \in E, x \neq 0$,

$$
1<1+1 / 4 c \leqq \phi(x) / x \leqq 1+1 / c \leqq 2,
$$

where $c+1$ is the first denominator of the simple continued fraction for $x$. We now write the second fraction on the right of (10) in the form

$$
\frac{Q_{n-1}}{q_{n-1}} \frac{c_{n}+1+s_{n}+t_{n-1}}{c_{n}+\phi\left(s_{n}\right)+\phi\left(t_{n-1}\right)},
$$

where

$$
\begin{aligned}
t_{n-1} & \equiv\left\{c_{n-1}+1, \cdots, c_{2}+1, c_{1}+1\right\}=Q_{n-2} / Q_{n-1}, \\
\phi\left(t_{n-1}\right) & =\left\{c_{n-1}, \cdots, c_{2}, c_{1}\right\}=q_{n-2} / q_{n-1},
\end{aligned}
$$


when $n>1$, and $t_{0}=\phi\left(t_{0}\right)=0$. Obviously, $t_{n-1} \in E$ for $n \geqq 1$. Applying the "outer" estimates of (12) to $\phi\left(s_{n}\right)$ and $\phi\left(t_{n-1}\right)$ and using $0 \leqq s_{n}+t_{n-1} \leqq 1$, we see that the second fraction in (13) is always bounded between 1 and 2. Treating the third fraction on the right of (10) similarly, we obtain from (10)

$$
G_{n}\left(Q_{n-1} / q_{n-1}\right)^{2} \leqq \Delta\left(x, x_{0}\right) \leqq 4 G_{n}\left(Q_{n-1} / q_{n-1}\right)^{2},
$$

where

$$
G_{n} \equiv\left(c-c_{n}+\phi(s)-\phi\left(s_{n}\right)\right) /\left(c-c_{n}+s-s_{n}\right) .
$$

We shall now state our results, concerning the derivatives of $\phi(x)$. We shall use the notations $D_{-}, D^{-}, D_{+}, D^{+}$for the lower and upper left-hand, lower and upper right-hand derivatives, respectively. If all four are equal we shall use the notation $\phi^{\prime}(x)$. We remark that, in investigating the behavior of the difference quotient $\Delta\left(x, x_{0}\right)$ as $x \rightarrow x_{0}$, where $x_{0} \in E$, we may restrict also $x$ to values of $E$, since for $x \in D$, $\Delta\left(x, x_{0}\right)$ lies between $\Delta\left(\xi, x_{0}\right)$ and $\Delta\left(\xi^{\prime}, x_{0}\right)$, where $\xi$ and $\xi^{\prime}$ are the end points of the $D_{n}$-interval to which $x$ belongs.

(4.1) When $x \in D$ then $\phi^{\prime}(x)=0$.

This follows at once from (3.1).

(4.2) $\phi^{\prime}(0)=1$.

By (2a) we have to show that $\Delta(x, 0)=\phi(x) / x \rightarrow 1$, as $x \rightarrow 0+$. We write $x$ in the form $x=\{c+1+s\}$ (see above). When $x \rightarrow 0+, c \rightarrow+\infty$, while $0 \leqq s \leqq 1 / 2$; thus our statement follows from (11).

(4.3) When $x_{0} \in E_{R}$ and $x_{0}$ is the right-hand end point of a $D_{n}$-interval $(n \geqq 2)$ we have $D_{-} \phi\left(x_{0}\right)=D^{-} \phi\left(x_{0}\right)=0$, while $D_{+} \phi\left(x_{0}\right)=D^{+} \phi\left(x_{0}\right)=(Q / q)^{2}$, where $Q$ and $q$ are the denominators of the reduced fractions for $x_{0}$ and $\phi\left(x_{0}\right)$, respectively. When $x_{0}$ is the left-hand end point of a $D_{n}$-interval $(n \geqq 1)$ the above relations hold with the indices - and + interchanged. (For examples, see the table at the end of the paper.)

Let $x_{0}=\left\{c_{1}+1, c_{2}+1, \cdots, c_{n}+1\right\}$ and assume that $x_{0}$ is the righthand end point of an interval of constancy of $\phi(x)$, that is, that $n$ is even. The first statement of (4.3) follows at once from (3.1). To investigate $\lim \Delta\left(x, x_{0}\right)$ as $x \rightarrow x_{0}+$, we may restrict $x$ to numbers of $E$ in the interval $x_{0}<x<\left\{c_{1}+1, c_{2}+1, \cdots, c_{n-1}+1, c_{n}+2\right\}$. Such an $x$ can be written in the form (9) with $c=c_{n}$ and $s \in E, s \neq 0$. By (6) we have in this case $s_{n}=0$. Substituting these values of $c$ and $s_{n}$ in (10) and using the reduction formulas for the $Q_{m}$ and $q_{m}$ (see $[8$, p. 28, (6) and (7) ]), we obtain

$$
\Delta\left(x, x_{0}\right)=\frac{\phi(s)}{s} \frac{Q_{n}}{q_{n}} \frac{\left(c_{n}+1+s\right) Q_{n-1}+Q_{n-2}}{\left[c_{n}+\phi(s)\right] q_{n-1}+q_{n-2}} .
$$


As $x \rightarrow x_{0}+$, we have $s \rightarrow 0+$ and hence, by (4.2), $\Delta\left(x, x_{0}\right) \rightarrow\left(Q_{n} / q_{n}\right)^{2}$ $=(Q / q)^{2}$, since $x_{0}=P_{n} / Q_{n}$ and $\phi\left(x_{0}\right)=p_{n} / q_{n}$. The proof for the lefthand end point of an interval of constancy of $\phi(x)$ requires only the changing of a few symbols in the above proof.

The result of (4.3) represents an interesting difference between our function $\phi(x)$ and the functions $\omega_{\alpha \beta}(x)$ of [3]. For the latter the derivative at the points of the corresponding sets $P^{-}$and $P^{+}$is zero on one side, but $+\infty$ on the other side. See [3, p. 438, Theorem 3$]$.

Before considering the case $x_{0} \in E_{I}$, we shall prove the following lemma, concerning the fractions $Q_{n} / q_{n}$, which in this case are defined for all positive integral $n$.

LEMMA. When $x_{0} \in E_{I}$ the fractions $Q_{n} / q_{n}$ form an increasing sequence which approaches a finite limit $L>1$ or $+\infty$, according to whether the infinite series

converges or diverges.

$$
\sum_{j=1}^{\infty} 1 / c_{j}
$$

We have $Q_{1} / q_{1}=1+1 / c_{1}$. Let $j>1$. Then by $(14),\left(Q_{j} / q_{j}\right) /\left(Q_{j-1} / q_{j-1}\right)$ $=\left(q_{j-1} / q_{j}\right) /\left(Q_{j-1} / Q_{j}\right)=\phi\left(t_{j}\right) / t_{j}$, and hence, by the "inner" estimates in $(12)$,

$$
1+1 / 4 c_{j} \leqq\left(Q_{j} / q_{j}\right) /\left(Q_{j-1} / q_{j-1}\right) \leqq 1+1 / c_{j} .
$$

Multiplying over $j$ from $j=2$ to $j=n$, we obtain for $n=1,2, \cdots$ $\prod_{j=1}^{n}\left(1+1 / 4 c_{j}\right)<Q_{n} / q_{n} \leqq \prod_{j=1}^{n}\left(1+1 / c_{j}\right)$. From these inequalities the lemma follows easily.

(4.4) Let $x_{0}=\left\{c_{1}+1, c_{2}+1, \cdots\right\} \in E_{I}$.

(i) If the $c_{j}$ are bounded then $\phi^{\prime}\left(x_{0}\right)=+\infty$.

(ii) If the $c_{j}$ are unbounded but the series (17) diverges, then ${ }^{7} D^{-} \phi\left(x_{0}\right)$ $=D^{+} \phi\left(x_{0}\right)=+\infty$.

(iii) If the series (17) converges then $D^{-} \phi\left(x_{0}\right)$ and $D^{+} \phi\left(x_{0}\right)$ are finite numbers greater than unity, while $D_{-} \phi\left(x_{0}\right)=D_{+} \phi\left(x_{0}\right)=0$.

Let $x \in E, x \neq 0, x \neq x_{0}$, and let the simple continued fractions for $x$ and $x_{0}$ agree in the first $n-1$ denominators but no further. We can then ${ }^{8}$ write $x$ in the form (9) with $c \neq c_{n}$ and make use of (15). We ob-

${ }^{7}$ No statement is being made about $D_{-} \phi\left(x_{0}\right)$ and $D_{+} \phi\left(x_{0}\right)$ in this case. In fact, this is the only problem, concerning the derivatives of $\phi(x)$, not answered in this section.

8 There is one exceptional case: the convergents of $x_{0}$, that is, the numbers $x=\left\{c_{1}+1, c_{2}+1, \cdots, c_{n-1}+1\right\}, n=2,3, \cdots$, cannot be written in the form (9) with $c \neq c_{n}$ and $s \in E$. A moment's consideration shows that their omission (as well as that of $x=0)$ from the values of $x$ in $\Delta\left(x, x_{0}\right)$ has no influence on the behavior of $\Delta\left(x, x_{0}\right)$ as $x \rightarrow x_{0}-$ or $x \rightarrow x_{0}+$. 
serve that $x \rightarrow x_{0}$ means that $n \rightarrow \infty$, while $c$ in (9) may assume any positive integral value, other than $c_{n}$, and $s$ in (9) any value of $E$. Finally, if $c>c_{n}$ we have $x>x_{0}\left(x<x_{0}\right)$ when $n$ is even (odd), while if $c<c_{n}$ the situation is reversed.

In the case (i), the series (17) diverges, hence, by the lemma, $Q_{n} / q_{n} \rightarrow+\infty$. Therefore, by (15) it will suffice to show that $G_{n}$ is greater than a positive constant for all values of $n, c$ and $s$ (see above). Since the $c_{j}$ are bounded and in view of (5), there exists a $\delta, 0<\delta$ $<1 / 4$, such that $\delta<s_{n}<1 / 2-\delta$ for all $n$, and hence by (3.2) an $\epsilon$, $0<\epsilon<1 / 2$, such that $\epsilon<\phi\left(s_{n}\right)<1-\epsilon$ for all $n$. Thus the denominator of $G_{n}$ (see (16)) is numerically less than $\left|c-c_{n}\right|+1 / 2$, while its numerator is numerically greater than $\left|c-c_{n}\right|-(1-\epsilon)$. In view of $\left|c-c_{n}\right| \geqq 1$, this shows that $G_{n}>2 \epsilon / 3$.

In the case (ii) we still have $Q_{n} / q_{n} \rightarrow+\infty$. Hence in view of (15), we have only to show the existence of two sequences of numbers in $E$, approaching $x_{0}$, one from the left and one from the right, such that $G_{n}$ for all members of these sequences is greater than a positive constant. These sequences are obtained by putting $c=c_{n}+1$ and $s=s_{n}$ for each $n$, in which case (16) gives $G_{n}=1$. And for even (odd) $n$ we have approach from the right (left).

In the case (iii), $Q_{n} / q_{n}$ approaches a finite limit $L>1$ (see the lemma). In order to show that $D^{-} \phi\left(x_{0}\right)$ and $D^{+} \phi\left(x_{0}\right)$ are finite, it suffices by (15) to show that $G_{n}$ is bounded above by a constant for all $n, c$ and $s$. But it is easily seen that the denominator of $G_{n}$ in (16) is numerically greater than or equal to $\left|c-c_{n}\right|-1 / 2$, its numerator is numerically less than or equal to $\left|c-c_{n}\right|+1$ and hence $G_{n} \leqq 4$. In order to show that $D^{-} \phi\left(x_{0}\right)$ and $D^{+} \phi\left(x_{0}\right)$ are greater than unity, we use the method used above in case (ii). In fact, the same sequences, defined by $c=c_{n}+1, s=s_{n}$, hence $G_{n}=1$, will suffice here. ${ }^{9}$ Finally, in order to show that $D_{-} \phi\left(x_{0}\right)=D_{+} \phi\left(x_{0}\right)=0$, we remark that by (3.2) these derivatives could not be less than zero and hence the method of case (ii) can again be used, only that here (see (15)) we have to choose $c$ and $s$ in such a way that $G_{n} \rightarrow 0$. Since, in case (iii), $c_{n} \rightarrow \infty$ and hence, by (5), $s_{n} \rightarrow 0+$, we may put (for sufficiently large $\left.n\right) c=c_{n}-1$ and $s=1 / 2$, so that, by (16), $G_{n}=\phi\left(s_{n}\right) /\left(1 / 2+s_{n}\right) \rightarrow 0$, as $n \rightarrow \infty$. This completes the proof of (4.4).

We remark that the cases (i), (ii) and (iii) of (4.4) divide the set $E_{I}$ into three subsets, each of which has the power of the continuum.

${ }^{9} \mathrm{By}$ the use of more careful estimates it is not difficult to show that $D^{-} \phi\left(x_{0}\right)$ $=D^{+} \phi\left(x_{0}\right)=2 L^{2}$, where $L=\lim _{n}\left(Q_{n} / q_{n}\right)$. The details of the proof of this fact are not given here. 
We have thus exhibited a continuum of points at which ${ }^{10} \phi^{\prime}(x)=+\infty$ (case (i)) and also one at which neither the left-hand nor the righthand derivative exists (case (iii)). These two subsets correspond to the types I and IV, respectively, of [3, p. 442].

The methods used in this section can also be applied to the problem of whether the function $\phi(x)$ satisfies a Lipschitz condition: $\phi\left(x^{\prime}\right)$ $-\phi(x) \leqq M\left(x^{\prime}-x\right)^{\nu}$ for $0 \leqq x<x^{\prime} \leqq 1$. (Compare [3, p. 437, Theorem 2].) We mention without proof that $\phi(x)$ satisfies a Lipschitz condition of order $\nu=\log b / \log a=.546$, where $a=\{2,2, \cdots\}=2^{1 / 2}-1$ and $b=\phi(a)=\{1,1, \cdots\}=\left(5^{1 / 2}-1\right) / 2$, but that $\phi(x)$ does not satisfy a Lipschitz condition of any higher order. The "best" value for the constant $M$, if $\nu=\log b / \log a$, is $10^{\nu / 2}=1.758$.

5. Table. The following table, which we give for the convenience of the reader, contains a few examples of $D_{n}$-intervals, which we denote here by $\left(x_{1}, x_{2}\right)$; it gives the value of $\phi(x)$ for each of these intervals and the left-hand (right-hand) derivative at its left (right) end point. The values of $x$ and $\phi(x)$ are written as simple continued as well as ordinary fractions.

TABLE

\begin{tabular}{|c|c|c|c|c|c|}
\hline \multirow{2}{*}{$n$} & \multicolumn{2}{|c|}{$D_{n}$-interval $=\left(x_{1}, x_{2}\right)$} & \multirow{2}{*}{$\begin{array}{c}\phi(x) \text { for } \\
x_{1} \leqq x \leqq x_{2}\end{array}$} & \multirow{2}{*}{$\begin{array}{l}D_{-} \phi\left(x_{1}\right) \\
D^{-} \phi\left(x_{1}\right)\end{array}$} & \multirow{2}{*}{$\begin{array}{l}D_{+} \phi\left(x_{2}\right) \\
D^{+} \phi\left(x_{2}\right)\end{array}$} \\
\hline & $x_{1}$ & $x_{2}$ & & & \\
\hline 1 & $\{2\}=1 / 2$ & $\{1\}=1$ & $\{1\}=1$ & 4 & - \\
\hline 2 & $\{3\}=1 / 3$ & $\{2,2\}=2 / 5$ & $\{2\}=1 / 2$ & $9 / 4$ & $25 / 4$ \\
\hline 2 & $\{4\}=1 / 4$ & $\{3,2\}=2 / 7$ & $\{3\}=1 / 3$ & $16 / 9$ & $49 / 9$ \\
\hline 2 & $\{5\}=1 / 5$ & $\{4,2\}=2 / 9$ & $\{4\}=1 / 4$ & $25 / 16$ & $81 / 16$ \\
\hline 3 & $\{2,2,2\}=5 / 12$ & $\{2,3\}=3 / 7$ & $\{1,2\}=2 / 3$ & 16 & $49 / 9$ \\
\hline 3 & $\{2,3,2\}=7 / 16$ & $\{2,4\}=4 / 9$ & $\{1,3\}=3 / 4$ & 16 & $81 / 16$ \\
\hline 3 & $\{3,2,2\}=5 / 17$ & $\{3,3\}=3 / 10$ & $\{2,2\}=2 / 5$ & $289 / 25$ & 4 \\
\hline 4 & $\{2,2,3\}=7 / 17$ & $\{2,2,2,2\}=12 / 29$ & $\{1,1,2\}=3 / 5$ & $289 / 25$ & $841 / 25$ \\
\hline
\end{tabular}

${ }^{10}$ In this connection, see [5, p. 258 , footnote 2]. We also note that all quadratic irrationalities between 0 and 1 fall under case (i). 


\section{BIBLIOGRAPHY}

1. F. Bernstein, Über eine Anwendung der Mengenlehre auf ein aus der Theorie der säkularen Störungen herribhrendes Problem, Math. Ann. vol. 71 (1912) pp. 417-439.

2. C. Carathéodory, Vorlesungen ibber reelle Funktionen, Leipzig and Berlin, 1927.

3. R. E. Gilman, A class of functions continuous but not absolutely continuous, Ann. of Math. (2) vol. 33 (1932) pp. 433-442.

4. F. Herzog and B. H. Bissinger, A generalization of Borel's and F. Bernstein's theorems on continued fractions, Duke Math. J. vol. 12 (1945) pp. 325-334.

5. E. Hille and J. D. Tamarkin, Remarks on a known example of a monotone continuous function, Amer. Math. Monthly vol. 36 (1929) pp. 255-264.

6. E. W. Hobson, The theory of functions of a real variable, vol. 1, Cambridge, 1927.

7. G. Kowalewski, Grundzilge der Differential- und Integralrechnung, Leipzig and Berlin, 1928.

8. O. Perron, Die Lehre von den Kettenbriichen, Leipzig and Berlin, 1929.

Michigan State College and

LEOMinster, MASS. 